\title{
INFLAMMATORY MYOFIBROBLASTIC TUMOR IN ADULT: A RARE CASE
}

\author{
Rajiv Nakarmi, ${ }^{1}$ Ming-Jenn Chen, ${ }^{2}$ Khaa- Hoo Ong, ${ }^{2}$ Muza Shrestha, ${ }^{1}$ Sundar Maharjan ${ }^{1}$
}

${ }^{1}$ Department of General and GI Laparoscopic Surgery, Manmohan Memorial Medical College and Teaching Hospital, Swyambhu, Kathmandu, Nepal, ${ }^{2}$ Chi Mei Medical Center, Tainan City, Taiwan

\begin{abstract}
Inflammatory myofibroblastic tumor (IMT) is a rare neoplasm of intermediate biologic potential. Inflammatory myofibroblastic tumor (IMT) of the alimentary tract often occurs in children or young adults, but may occur at any age. The lung is the most commonly affected location. However, it may appear in small bowel mesentery especially the distal ileum, mesotransverse colon, or greater omentum. A 35 years old female presented to out-patient department with complaint of mass in right abdomen since 1 month associated with loss of weight. Computed tomography of abdomen revealed a solid intraperitoneal mass arising from bowel mesentery. Laparoscopic excision of the tumor was performed and the histopathological examination of the mass revealed it to be an inflammatory myofibroblastic tumor arising from the omentum and large bowel mesentery. The post- operative period was uneventful with no evidence of tumor recurrence at follow up at 2 years. Inflammatory myofibroblastic tumor is a rare soft tissue tumor usually arising from lungs but tumor arising from the omental-mesenteric origin has also been documented. The precise diagnosis is made only by the histopathological evidence. Surgical resection is the treatment of choice.
\end{abstract}

\section{KEYWORDS}

Inflammatory Myofibroblastic tumor, mesentery, anaplastic lymphoma Kinase

Received on: March 14, 2021

Accepted for publication: July 16, 2021

\section{CORRESPONDING AUTHOR}

Dr. Rajiv Nakarmi

Junior Consultant,

Department of General and GI laparoscopic Surgery

Manmohan Memorial Medical College and Teaching

Hospital, Swoyambhu, Kathmandu

Email: rajivnakarmi31@gmail.com

Orcid No: https://orcid.org/0000-0003-2205-1784

DOI: https://doi.org/10.3126/nmcj.v23i4.42279 


\section{INTRODUCTION}

Inflammatory myofibroblastic tumors (IMT) are rare soft tissue tumors. ${ }^{1}$ It usually occurs in children and young adults. ${ }^{2}$ The IMT is a member of a heterogeneous group of soft tissue tumors. IMT have various names, including inflammatory pseudotumor, plasma cell granuloma, fibrous histiocytoma, solitary mast cell tumor, and fibroxanthoma. ${ }^{3}$ The clinical behavior of IMT is similar to that of tumors of uncertain malignant potential, so IMT is considered tumors of borderline malignancy. ${ }^{4}$ Most frequently involved organ is the lung, however intra-abdominal organs have also been involved such as small bowel, mesentery and omentum. ${ }^{5-7}$ Small bowel tumors are particularly rare, and even more rarely they are located in the antimesenteric edge of the small bowel. ${ }^{7}$

\section{CASE REPORT}

A 35 years old female had a history of bulge in right upper abdomen of 1 month duration. She noticed it while changing her clothes which was

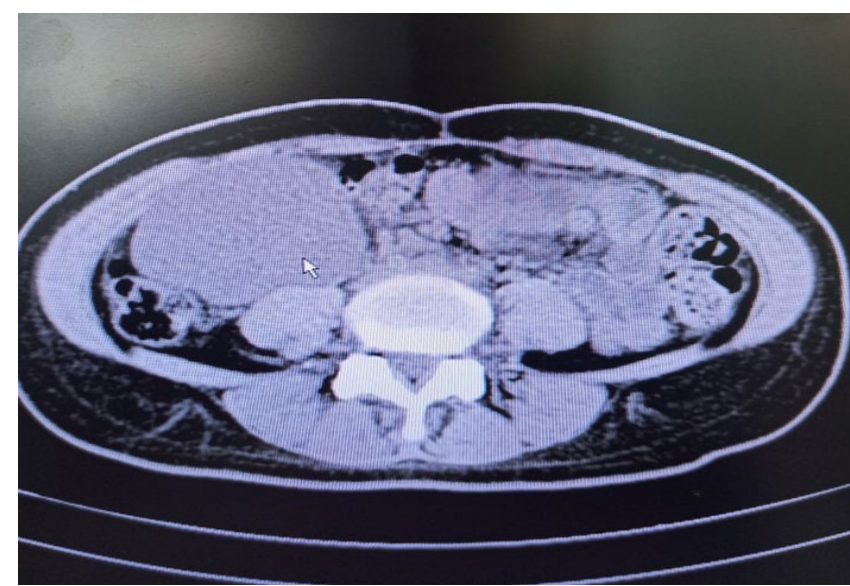

Fig. 1: CT abdomen showing tumor arising from right side of the abdomen initially small in size. The size of the mass had gradually increasing and was associated with weight loss of around $2-3 \mathrm{~kg}$ in the following month. She had no any other significant history.

Clinical examination revealed a solitary, spherical, smooth mass at right upper abdomen which was intraperitoneal. The size was around $8 * 5 * 3 \mathrm{~cm}$ and with no other significant findings on inspection. The mass was nontender, firm to hard in consistency.

Laboratory parameters were normal. Computed tomography showed a soft tissue lesion at the right mid and lower abdomen around $8.2 \mathrm{~cm}$ in size (Fig. 1) and small bowel was pushed towards the left side. Small bowel contrast series showed no mass lesion arising from the small bowel and the bowel was deviated to the left side of the abdomen, which was compatible with the CT findings of external compression by the tumor at the RLQ of the abdomen. Provisional Diagnosis of GIST was made.

She was taken up for Diagnostic laparoscopy and proceed. Intraoperative findings were:

- There was a tumor of $11 * 9 * 7 \mathrm{~cm}$ arising from the proximal transverse colon also around hepatic flexure which was hard in consistency (Fig.2).

- Omentum was adherent to the tumor and there were multiple small nodular lesions over the omentum.

- There were no ascites, liver and peritoneum was normal.

Laparoscopic tumor excision with segmental colectomy was done and the specimen was send for examination. The Histopathological examination of the specimen showed inflammatory myofibroblastic tumor arising from the mesentery and omentum. The sections showed a circumscribed tumor with focal infiltration into the adjacent fat and composed

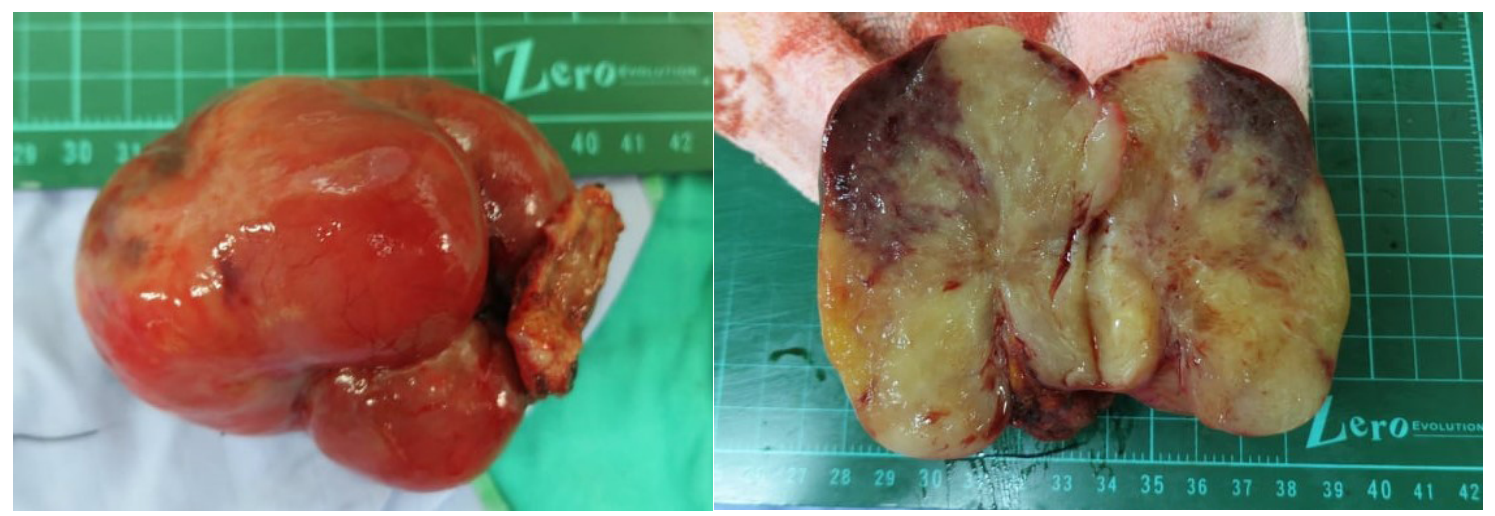

Fig. 1: Showing the excised tumor 
of spindled or epithelioid myofibroblasts in a myxoid and sclerotic background with abundant capillary sized vessels admixed with infiltration of plasma cells and lymphocytes. Immunohistochemically the tumor cells showed distinctive nuclear membrane staining ALK (anaplastic lymphoma kinase). Positive staining for smooth muscle actin. CD117 and cdk-4 (cyclin-dependent kinase) staining were negative. Post-operative period was uneventful and during follow up CT scan no any tumor recurrence was noted even after 2 years of surgery.

\section{DISCUSSION}

Dr. Brunn had first described inflammatory myofibroblastic tumor in 1939. ${ }^{8}$ IMT is a rare lesion that belongs to the group of soft tissue tumors. The condition is also termed as inflammatory pseudotumor, plasma cell granuloma, fibrous histiocytoma, solitary mast cell tumor, and fibroxanthoma. ${ }^{3}$ The most common affected organ reported was lung. ${ }^{9,10}$ However, IMT can occur at any organ of the body. Among extrapulmonary IMTs, $43 \%$ were found arising from the mesentery and omentum.5,11 Mesentery of small bowel, especially at distal ileum, transverse colon, and great omentum were the most common affected sites. ${ }^{1}$ In this case the tumor was found arising from the large bowel mesentery and omentum.

The etiologic factors responsible for the development of IMT are not clearly established., ${ }^{3,6}$ IMT may represent an immunologic response to an infectious or noninfectious agent, or it may be a true tumor. Campylobacter jejuni, Epstein-Barr virus, and Escherichia coli have been associated with IMT. Also, trauma, steroid use, abdominal surgery, and genetic factors have been reported, but the pathogenesis of IMT remains unclear. Presenting symptoms depend on the involved primary site by the tumor. The lung is the most commonly affected site, but extrapulmonary IMT may include the mesentery-omentum, upper respiratory tract, genitourinary tract, retroperitoneum, pelvis, head, neck, spleen, brain, pancreas, liver, and gastrointestinal tract. ${ }^{4,6}$ Documented IMT cases in the mesentery-omentum have been located mostly in the mesentery of the small bowel and not in the antimesenteric edge. ${ }^{7}$ This case report also showed tumor arising from the mesenteric end. Patients with intraabdominal tumors most commonly present with intermittent abdominal pain due to the solid mass, weight loss, as well as abdominal distention, malaise, anorexia, and vomiting. ${ }^{3,7,1,12}$ Abdominal pain was not evident in our case. Rarely, the presentation may be complicated by an intestinal obstruction, intussusception, or acute abdomen mimicking acute appendicitis, as was the case in our patient. $^{2}$ Occasionally, IMT presented with symptoms like acute abdomen. ${ }^{13}$

Laboratory abnormalities are present in a minority of patients and include an elevated erythrocyte sedimentation rate (ESR), anemia, thrombocytosis, or hypergammaglobulinemia, which often resolve after resection. ${ }^{14-17}$ However these laboratory parameters were not evident in this case.

There is no specific findings in imaging studies and is often diagnosed preoperatively as other intra-abdominal tumors, such as sarcoma, lymphoma, adenocarcinoma, GIST, desmoid tumor, or carcinoid tumor. ${ }^{1,5}$ Because most of the IMTs has extensive central necrosis ${ }^{[18]}$, it is important to include IMT in the differential diagnosis of any mesenteric mass that contained large, irregular areas of necrosis. Also mesenteric or omental IMF tumors appeared as well defined solid, mixed-echogenic masses in sonography and prominent vascularity on Doppler sonography. On CT scans, these tumors shows typically heterogeneous attenuating enhancement ${ }^{19}$ as shown in Fig. 1. However, with these findings, definite radiologic differentiation of an IMF tumor from other malignancies is not definitive. Imaging studies may be helpful not for differentiation between benign and malignant lesion, but for the decision whether complete resection can be possible. The diagnosis of IMF tumor should be established with certainty only on pathological examination. On MRI, intraabdominal myofibroblastic tumors usually exhibit an intermediate signal intensity on T1-weighted images and a heterogeneous high signal intensity on T2- weighted images. ${ }^{20}$ On T1weighted images after Gd- PTA, intraabdominal IMT shows a variable enhancement pattern depending on the relative amount of fibrous tissue and cellular material. ${ }^{20}$ Thus, MRI can be considered additional modality for further differential diagnosis of mesenteric mass containing large, irregular areas of necrosis.

The diagnosis is usually after the histopathological evidence. Spindled myofibroblasts, and inflammatory cells are the most frequent cellular components of IMT. Tumors composed of myofibroblasts and fibroblasts pose significant challenges in differential diagnosis because of their morphological overlap with IMT. This group includes fibrous histiocytoma, malignant fibrous histiocytoma, nodular fasciitis, and other pseudosarcomas as fibromatosis, 
myofibroblastoma,

congenital-infantile fibrosarcoma, or conventional fibrosarcoma. ${ }^{21}$ Malignant fibrous histiocytoma are characterized by marked cytological and nuclear pleomorphism, often with bizarre tumor giant cells, admixed with spindle cells, and often rounded histiocyte like cells in varying proportions. ${ }^{2}$ By means of immunohistochemistry, malignant fibrous histiocytoma lack cytoplasmic reactivity for vimentin and smooth muscle actin. ${ }^{5}$ Extrapulmonary IMT are histologically categorized into three types ${ }^{1,18}$ :

a. Resemble granulation tissue, nodular fasciitis.

b. Resemble fibromatosis, fibrous histiocytoma, and smooth muscle neoplasm.

c. Resemble a scar or desmoid fibromatosis.

Performing immune-histochemical staining, most IMTs show cytoplasmic reactivity for vimentin and smooth muscle actin. ${ }^{5}$ Also, ALK positivity is detected in 36 to $60 \%$ of cases, and is associated with cytogenetic abnormalities involving the ALK gene on chromosome 2. The presence of chromosomal aberrations (30 to $40 \%$ of cases) in these tumors suggests that IMT is a neoplastic proliferation of clonal origin. ${ }^{22}$ In our case, there was no evidence of infection or inflammatory response and no history of trauma in her abdomen. The tumor was single, well circumscribed, and movable. The immunehistochemical staining result for ALK-1 shows strong positivity of tumor cytoplasm.

In contrast to malignant fibrous histiocytoma in gross appearance, IMT showed a circumscribed or multinodular firm, white, or tan mass with a whorled flesh or myxoid cut surface. Malignant fibrous histiocytoma showed well circumscribed, expansive masses which may appear pseudo capsulated, and cut surface is variable and may include pale fibrous or fleshy areas. ${ }^{[5]}$ In most reported cases, a solitary lesion was found for pulmonary or extrapulmonary IMT. However, multiple nodules might be occasionally present. The size reported ranged from $1 \mathrm{~cm}$ to $17 \mathrm{~cm} .^{23}$ In comparison, most extrapulmonary IMTs, especially those of the mesenteric type, were larger in size. ${ }^{23}$ Recurrence were documented in $18 \%$ to $40 \%$ of reported cases and appeared to occur more frequently in extrapulmonary IMTs, which were often larger than $8 \mathrm{~cm}$ and locally invasive in primary tumor. ${ }^{23,24}$ However in this case there was no evidence of recurrence even at 2 years of surgery.

The treatment is straight forward; resection remains to be the best method of choice. Some other treatments such as adjuvant chemotherapy or radiotherapy have been tried in the past. ${ }^{23}$ Unfortunately, all of these adjuvant treatments of IMT were proved to have no or only little benefit for the patient. ${ }^{17}$

IMT is a rare soft tissue tumor which usually occurs in children but also can be seen in adults. Intra-abdominal IMTs are clinically and radiologically difficult to differentiate from other intraabdominal malignant tumors. Laboratory investigations may not be reliable as it is seen only in minority of cases. In an elderly patient with an abdominal mass, it is reasonable to consider the possibility of IMT if other diagnoses are excluded. MRI might provide useful information for diagnosis preoperatively. Since IMTs of the alimentary tract have a high potential of local recurrence, and treatments are not effective, aggressive en-bloc resection of the tumors remains the best choice of treatment for patients with IMTs. Furthermore, in case that the tumor is larger than $8 \mathrm{~cm}$, multinodular, originated from omentum, ill-defined in margin, or presented with pathologically atypia, ganglion like cells, a close follow-up after primary surgery might be necessary for an early detection of a recurrent tumor.

\section{Conflict of interest: None}

Source of research fund: None

\section{REFERENCES}

1. Bonnet JP, Basset T, Dijoux D. Abdominal inflammatory myofibroblastic tumors in children: report of an appendiceal case and review of the literature. J Pediatr Surg 1996; 31: 1311-4.

2. Karnak I, Senocak ME, Ciftci AO et.al. Inflammatory myofibroblastic tumor in children: diagnosis and treatment. J Pediatr Surg 2001; 36: 908-12.

3. Dhouib A, Barrazzone C, Reverdin A, Anooshiravani M, Hanquinet S. Inflammatory myofibroblastic tumor of the lung: a rare cause of atelectasis in children. Pediatr Radiol 2013; 43: 381-4.

4. Koyuncuer A. Inflammatory myofibroblastic tumor of the small-bowel mesentery: A case report of nonspecific clinical presentation and a review of the literature. Int'l J Surg Case Rep 2014; 5: 1214-7.

5. Coffin CM, Hornick JL, Fletcher CD. Inflammatory myofibroblastic tumor: comparison of clinicopathologic, histologic, and immunohistochemical features including ALK 
expression in atypical and aggressive cases. $A m$ J Surg Pathol 2007; 31: 509-20.

6. Appak YÇ, Sahin GE, Ayhan S, Taneli C, Kasırga E. Inflammatory myofibroblastic tumor of the colon with an unusual presentation of intestinal intussusception. European J Pediatr Surg Rep 2014; 2: 54-7.

7. Difiore JW, Goldblum JR. Inflammatory myofibroblastic tumor of the small intestine. J Am Coll Surg 2002; 194: 502-6.

8. Brunn H. Two interesting benign lung tumors of contradictory histopathology. J Thorac Surg 1939; 9: 119-31.

9. Bahadori M, Liebow AA. Plasma cell granulomas of the lung. Cancer 1973; 31: 191-208.

10. Pettinato G, Manivel JC, De Rosa N, Dehner LP. Inflammatory myofibroblastic tumor (plasma cell granuloma). Clinicopathologic study of 20 cases with immunohistochemical and ultrastructural observations. Am J Clin Pathol 1990; 94: 538-46.

11. Khoddami M, Sanae S, Nikkhoo B. Rectal and appendiceal inflammatory myofibroblastic tumors. Arch Iran Med 2006; 9: 277-81.

12. Alaggio R, Cecchetto G, Bisogno $G$.et.al. Inflammatory myofibroblastic tumors in childhood: a report from the Italian Cooperative Group studies. Cancer 2010; 116: 216-26.

13. Heim D, Ruchti C, Negri M. Acute abdomen caused by a perforated inflammatory myofibroblastic tumor of the jejunum. Int'l Surg 2006; 91: 63-7.

14. Donner LR, Trompler RA, White RR 4th. Progression of inflammatory myofibroblastic tumor (inflammatory pseudotumor) of soft tissue into sarcoma after several recurrences. Hum Pathol 1996; 27: 1095-8.

15. Stringer MD, Ramani P, Yeung CK, Capps SN, Kiely EM, Spitz L. Abdominal inflammatory myofibroblastic tumours in children. Br J Surg 1992; 79: 1357-60.
16. Souid AK, Ziemba MC, Dubansky AS, et al. Inflammatory myofibroblastic tumor in children. Cancer 1993; 72: 2042-8.

17. Sanders BM, West KW, Gingalewski C, Engum S, Davis M, Grosfeld JL. Inflammatory pseudotumor of the alimentary tract: clinical and surgical experience. J Pediatr Surg 2001; 36: 169-73.

18. Apa H, Diniz G, Saritas T, et al. Abdominal inflammatory myofibroblastic tumor: review of the literature by means of a case report. Turkish J Cancer 2002; 32: 28-31.

19. Kim SJ, Kim WS, Cheon JE et al. Inflammatory myofibroblastic tumors of the abdomen as mimickers of malignancy: imaging features in nine children. Am J Roentgenol 2009; 193: 141924.

20. Ko SW, Shin SS, Jeong YY. Mesenteric inflammatory myofibroblastic tumor mimicking a necrotized malignant mass in an adult: case report with MR findings. Abdom Imaging 2005; 30: 616-9.

21. Coffin CM, Dehner LP, Meis-Kindblom JM. Inflammatory myofibroblastic tumor, inflammatory fibrosarcoma, and related lesions: an historical review with differential diagnostic considerations. Semin Diagn Pathol 1998; 15: 102-10.

22. Saleem MI, Ben-Hamida MA, Barrett AM, et.al. Lower abdominal inflammatory myofibroblastic tumor -an unusual presentation- a case report and brief literature review. Eur J Pediatr 2007; 166: 679-83.

23. Coffin CM, Watterson J, Priest JR, Dehner LP. Extrapulmonary inflammatory myofibroblastic tumor (inflammatory pseudotumor). A clinicopathologic and immunohistochemical study of 84 cases. Am J Surg Pathol 1995; 19: 85972 .

24. Dao AH, Hodges KB. Inflammatory pseudotumor of the pelvis: case report with review of recent developments. Am Surg 1998; 64: 1188-91. 